\title{
Large Aperture and Wedged Multilayer Laue Lens for X-ray Nanofocusing
}

\author{
Nathalie Bouet ${ }^{1}$, Albert T. Macrander², Jörg Maser ${ }^{2}$, Yong S. Chu ${ }^{1}$, Juan Zhou ${ }^{1}$, Evgeny Nazaretski ${ }^{1}$, \\ Hanfei Yan ${ }^{1}$, Xiaojing Huang ${ }^{1}$, and Ray Conley ${ }^{1,2, *}$ \\ ${ }^{1}$ National Synchrotron Light Source II, Brookhaven National Laboratory, Upton NY 11973, USA \\ ${ }^{2}$ Advanced Photon Source, Argonne National Laboratory, Argonne IL 60439, USA
}

\begin{abstract}
Diffraction optics fabricated from multilayers offer an intriguing alternative to lithography-based zone plates due to their advantages of virtually limitless aspect ratio and extremely small feature size. However, other issues, intrinsic to thin-film deposition, such as film stress and deposition rate instability, for example, limit the total achievable aperture. Over the last decade, Multilayer Laue Lens (MLLs) have progressed from a mere curiosity with initial aperture sizes in the 3-10 $\mu \mathrm{m}$ range, to real beamline-deployed optics with apertures in the 40-50 $\mu \mathrm{m}$ range (X. Huang, et al., Scientific Reports 3, 3562 (2013); E. Nazaretski, et al., Rev. Sci. Instrum. 85, 033707 (2014); E. Nazaretski, et al., Journal of Synchrotron Radiation 24, 1113 (2017)). By optimizing deposition conditions and incorporating new materials, MLLs have now broken the $100 \mu \mathrm{m}$ thickness milestone. A flat $\mathrm{WSi}_{2} / \mathrm{Al}-\mathrm{Si} \mathrm{MLL}$ with a deposition thickness of $102 \mu \mathrm{m}$, the largest MLL to date, is reviewed. New large aperture wedged MLLs (wMLL), which were first fabricated by APS in 2006 using the $\mathrm{WSi}_{2} / \mathrm{Si}$ material system, are presented which demonstrate high focusing efficiency across a broad energy range. These results confirm findings by other groups who have also independently fabricated wMLL (A. J. Morgan, et al., Scientific Reports 5, 9892 (2015); S. Bajt, et al., Nature Light: Science and Applications 7, 17162 (2017)) based on a similar material system.
\end{abstract}

Keywords: Multilayer, X-ray Optics, Multilayer Laue Lens, MLL, Nanofocusing, $\mathrm{WSi}_{2} / \mathrm{Si}, \mathrm{Al}-\mathrm{Si}$.

\section{INTRODUCTION}

Investigation of the internal structure of objects in applied fields and extreme environments is possible with X-rays due to their weak interaction with matter. Reflective, ${ }^{6}$ refractive $^{7}$ or diffractive ${ }^{8,9} \mathrm{X}$-ray nanofocusing optics are utilized in various applications for high-resolution imaging. Diffractive X-ray optics feature a high numerical aperture due to their geometry and present-day fabrication limitations, so there remains substantial promise to approach spatial resolutions currently only obtained by electron microscopy. A Fresnel Zone Plate (FZP) is comprised of a series of optically absorbing radially symmetric rings, or zones. By locating the Fresnel zones according to the FZP formula, light diffracting around each zone constructively interferes at the focus. The basic schematic of a flat FZP is depicted below, in Figure 1(a).

Lithographically produced FZP are widely used in both scanning probe and full-field imaging applications, ${ }^{9}$ due to their simple alignment, excellent layer placement

\footnotetext{
*Author to whom correspondence should be addressed.
}

precision, and essentially unlimited overall diameter. Optical thickness, or section depth, defines the optical efficiency because the maximum occurs when the layers provide a $\pi / 2$ phase shift. The spatial resolution of the optic is proportional to the width of the outermost zones, and efficiency is significantly improved when the individual zones are tilted (or curved) to satisfy the local Bragg condition.

Significant work on zone plate fabrication with advanced structures such as zone stacking, zone interlacing, zone doubling, and multi-level processing have yielded focusing performance of $7.8 \mathrm{~nm}$ focal size at $0.5 \%$ efficiency. ${ }^{10}$ State-of-the-art zone plates,${ }^{11-15}$ found in use today at beamlines, are lithographically produced with $20 \mathrm{~nm}$ outermost zone widths, and aspect ratios between 20 and 30. Although these perform extremely well, the section depth remains many times smaller than the requirements for optimum efficiency. As outermost zone width decreases, and energy increases, aspect ratios of 1,000:1 or greater are required.

Thin-film based fabrication techniques bypass these limitations because thin-films are deposited at sub-nm 

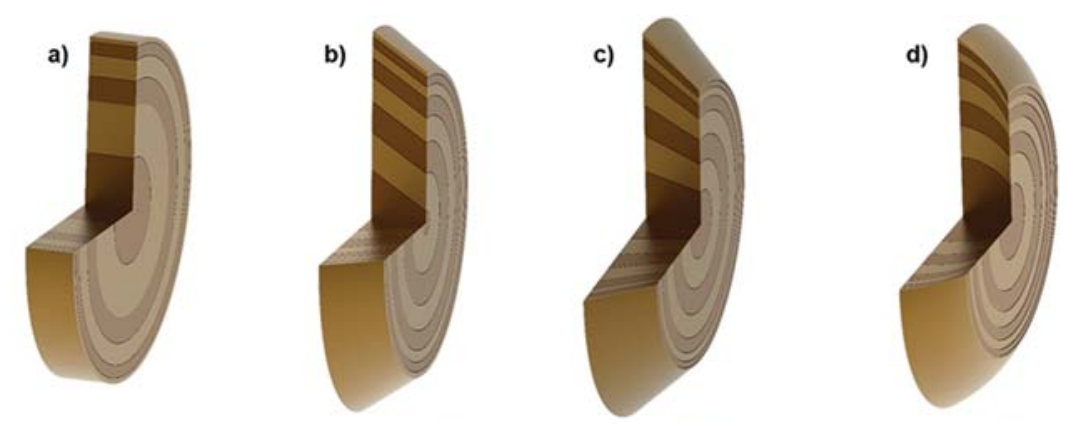

e)

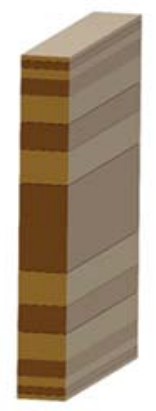

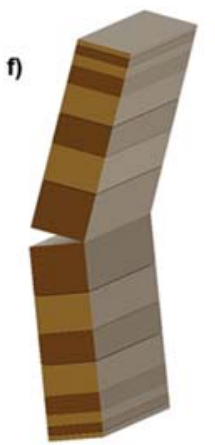

g)

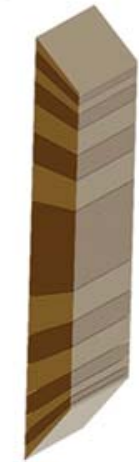

h)

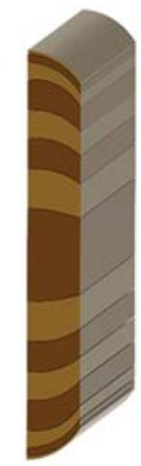

Figure 1. Various geometries for nanofocusing diffractive optics, both circular/radially symmetric (top row) and linear versions (bottom row). A conventional deposition-on-flat-wire optic is seen in (a) with axially uniform layering. This is the most geometrically similar multilayer-based structure to a conventional zone plate (albeit the lack of filler in a conventional zone plate provides clear optical advantage). The analogous linear structure with flat zones is seen in (e), directly below. Of the various configurations, flat structures offer the lowest efficiency. Part (b) depicts axially uniform deposition of layers onto a tapered wire, and the linear equivalent directly below in part (f) is achieved by tilting $1 / 2$ of a flat structure. Efficiency is greatly improved over untilted structures because a portion of the structures satisfy the Bragg condition. Axially graded layering on a tapered wire (c) or a flat substrate $(\mathrm{g})$ offers the highest efficiency because every zone in structure is properly tilted to satisfy the Bragg condition for most of the zones. Curved interfaces can be conceptualized in a radially symmetric fashion as a (roughly) elliptical profile deposited on a sectioned sphere, as in (d), guaranteeing Bragg condition for all zones. The linear equivalent can be obtained by depositing an elliptical profile onto a flat substrate that lies directly in the center of the optical axis ((a) double-structure is depicted in (h)).

thickness ranges, and sectioned to arbitrary section widths. With interfacial roughness and chemical intermixing defining the minimum achievable layer thickness, optics with zones well below $1 \mathrm{~nm}$ (and thus resolutions below $1 \mathrm{~nm}$ ) are plausible. This fact led to the early development of "jelly roll" zone plates, ${ }^{16-20}$ also known as "sputteredsliced zone plates" or "multilayer zone plates." These are shown schematically in Figure 1(a), where a multilayer is deposited around a wire and then sectioned to the proper width, providing maximum diffraction efficiency. Some recent successes with this method are reported. ${ }^{21-25}$ Variations from the uniform, radially symmetric structure can incorporate certain embodiments of layer tilt to increase efficiency. These include deposition of an axially uniform multilayer on a tapered core to achieve global tilting of all layers as in Figure 1(b), or deposition of axially graded multilayers on a tapered core to achieve a wedged structure as in Figure 1(c). Such a structure was fabricated in at least one case. ${ }^{23}$ Individually curved layers, only important at resolutions of perhaps below $0.7 \mathrm{~nm}$, are conceptualized via deposition of a multilayer with an axial gradient that is parabolic or elliptical in figure over a spherical substrate. Such a structure is shown in Figure 1(d).
While circular-wire multilayer zone plates would be highly appropriate optics to utilize as drop-in alternatives to conventional zone plates, many efforts ran into performance limits due to a combination of fabrication issues. Critically, the interfacial roughness must be kept low enough to maintain adequate contrast between adjacent layers. The physical vapor deposition techniques and materials utilized generate roughness during off-axis deposition. Since only a small frontal region of a round wire lies approximately normal to the source at any given point in time, roughness propagation was an intrinsic problem. Imperfect wire circularity displaces the zones, resulting in focus blurring and aberrations. Roughness and layer placement errors accumulate, progressively displacing the outermost zones which have the most sensitive effect on focal performance. However, some recent successes of on-wire deposition with alternative deposition techniques have led to renewed interest. ${ }^{21,25}$

These limitations led to development of a linear multilayer zone plate, called a Multilayer Laue Lens ${ }^{26}$ (MLL). Two primary changes in geometry are noted. The first, and main, difference is that, instead of starting with a round wire, the structure is deposited on a super-polished flat 
substrate which are readily available. The second difference is that the thinnest layers are deposited first. This avoids the buildup of accumulated roughness and layer imperfections from long depositions affecting the most sensitive thinnest layers. Later advancements in deposition system stability and process optimization are eliminating the need to fabricate the thinnest layers first; however, the use of high quality flat substrates continues to provide good results. The main disadvantage of MLLs is that two lenses, sectioned and aligned in a crossed geometry, are required to produce a point focus. A flat MLL is shown schematically in Figure 1(e). By tilting a flat MLL as depicted in Figure 1(f), the diffraction efficiency increases within a significant portion of the structure. Tilted (or flat) MLLs provide focal performances down to about $5 \mathrm{~nm}$ with good efficiency according to theoretical modeling $;{ }^{27-29}$ however, even smaller spatial resolution and higher efficiency can be obtained if every layer is tilted appropriately to the wedged geometry in Figure 1(g). A half MLL structure composed of curved layers (full structure shown for comparison in Fig. 1(h)) is also possible to fabricate by deposition of an elliptical (or parabolic) profile. This starts with a flat substrate located in the center of the optical axis or an elliptically figured substrate located near the outer region. Such a precise structure is only required to obtain a spatial resolution below about $0.7 \mathrm{~nm}$. Therefore, it is more important to take into consideration more immediate fabrication limitations such as layer placement precision, aperture limits, and bonding technology.

At their introduction, a little over a decade ago, ${ }^{30,31}$ MLL deposition limits were in the range of 11-12 $\mu \mathrm{m}$, producing focal sizes as small as $30 \mathrm{~nm}$ with efficiencies up to $44 \% .^{32}$ In 2008 , measurements at $19.5 \mathrm{keV}$ demonstrated a line focus of $16 \mathrm{~nm}$ width with an efficiency of $31 \%$ using a partial MLL structure with an outermost zone width of $5 \mathrm{~nm} .{ }^{33}$ This initial work focused on DC magnetron sputtering of tilted half-structures; however, some success was reported ${ }^{34}$ producing full MLL structures using a bonding technique to double the optical aperture. The difficult alignment of both halves is achieved by including a transverse thickness gradient in the structure $^{35}$ and selecting the region where the two halves properly match. This approach reduces sample yield because only one region in each structure produces a matching focal plane. The first wedge MLL (wMLL), where layer placement not only follows the FZP formula, but is also laterally-graded along the optical axis, was produced in $2006^{36}$ using a figured deposition-flux mask. Shortly thereafter, MLL efforts worldwide explored new fabrication techniques, including the use of alternative materials, ${ }^{37,38}$ new deposition processes (pulsed-laser deposition $^{39,40}$ ) as well as novel methods ${ }^{41-43}$ to incorporate lateral gradients for wMLL fabrication. A tilted MLL consisting of parallel layers was used to focus $12 \mathrm{keV}$ X-rays to a spot of $11.2 \mathrm{~nm}^{44}$ (FWHM) with $15 \%$ efficiency. Recently, Sub 10-nm ${ }^{5,21,45}$ focal performance were reported at multiple locations. A hard X-ray microscope with $11 \times 12 \mathrm{~nm}^{1-3}$ spatial resolution is in regular user operations at the National Synchrotron Light SourceII (NSLS-II). The optics in service have an aperture of $43 \mu \mathrm{m}$ and $53 \mu \mathrm{m}$, the largest aperture known to be reported at the time of their deployment. Here, we summarize results on a $102 \mu \mathrm{m}$ aperture MLL, about twice the largest previously reported. This large-aperture flat MLL is a first trial of a new material system, $\mathrm{WSi}_{2} / \mathrm{Al}-\mathrm{Si}$. Also, a series of wMLLs were fabricated using the $\mathrm{WSi}_{2} / \mathrm{Si}$ and $\mathrm{WSi}_{2} / \mathrm{Al}-\mathrm{Si}$ material systems and measured for efficiency at beamline 1BM at the APS. The large energy efficiency range observed confirms results from other groups who have also recently began fabricating wMLLs using similar materials and related shadow masking techniques, with impressive results. ${ }^{4,5}$

\section{LARGE APERTURE MLL OPTICS, REACHING THE $100 \mu \mathrm{m}$ MARK}

A small aperture MLL is of limited utility for beamline use, it is restricted to a short focal length and the small aperture fails to capture a significant portion of the incident radiation, resulting in low overall beamline efficiency and low focused flux. Large aperture MLL optics are required in order to maintain reasonably large focal lengths and capture a significant portion of the incident beam. MLL optics with a physical aperture larger than $100 \mu \mathrm{m}$ are highly desirable for this reason. However, achieving this goal requires solving substantial fabrication challenges. Film stress and inter-related structural robustness limitations are the most common source of deposition failure in thick films such as large aperture MLL. Accumulated film stress compromises the survival of the multilayer during growth as well as producing bulk defects that adversely affect the MLL's focusing performance. Thin film stress is usually produced independently of the method of deposition, and has been studied in depth. ${ }^{46-49}$ In the case of MLL, the challenge is not only to produce a structurally sound multilayer with an exceedingly large number of layers and total thickness, but also one where the interfacial width remains sufficiently low to retain high optical performance.

Significant studies around the world ${ }^{50}$ have investigated the interconnection that materials and deposition processes share in the formation of interfacial roughness and film stress during growth. Deposition processes contain a plethora of ions, electrons, and energetic neutrals, which add mobility ${ }^{51}$ that can dominate interfacial smoothening. ${ }^{52}$ The goal is generally to maintain as high an energy transfer as possible at the deposition surface for creating interfaces with minimal interfacial roughness, but not to concurrently bombard the surface with plasma from the deposition source which can destroy the growing multilayer. This kinetic bombardment also tends to lead to high 
film stress. ${ }^{53}$ Accumulated film stress within a sputterdeposited multilayer is typically highly compressive when the material is grown at low pressures ${ }^{54}$ and gradually decreases to stress-neutral or even tensile with a higher working-gas. Deposition of thin films at high process gas pressures allows the fabrication of MLLs with exceedingly large apertures; however, a study of film stress and pressure with the $\mathrm{WSi}_{2} / \mathrm{Si}$ material system ${ }^{55}$ reports that a pressure threshold of about 6 milliTorr was found to correspond with a significant increase in interfacial roughness. Further increases of pressure up to 12 milliTorr resulted in a stress-neutral state, but with even more pronounced waviness and layer disruption. Alternative deposition techniques have reported some successes. MLL fabricated from $\mathrm{Ti} / \mathrm{ZrO}_{2}$ using pulsed laser deposition were reported to exhibit a frequency-dependent smoothing of the rough surface, ${ }^{56}$ forming an MLL with very sharp and narrow interfaces. In order to double the aperture, two MLL halves can be bonded together. ${ }^{34}$ However, the two halves must be aligned to a spacing precision within $1 / 3$ of the outermost zone, and so this is only useful if an intentional gradient is introduced. ${ }^{57}$ An alternative to bonding in order to expand the aperture is to find ways to reduce accumulated film stress at pressures below 6 milliTorr to achieve larger deposition thicknesses. As the contribution to stress from individual material components is unequal, one proposed method of reducing the accumulated stress is to vary the bilayer ratio. ${ }^{58}$ However, this degrades optical efficiency, requiring a correspondingly larger aperture optic in order to regain total focused flux. The current process utilizes reactive sputtering (discussed below), and introduce new materials in order to break the $100 \mu \mathrm{m}$ deposition milestone.

The discovery of bulk defects within MLLs when deposition thicknesses reached the $40 \mu \mathrm{m}$ range led to work on reactive deposition ${ }^{59}$ with $\mathrm{N}_{2}$. This significantly reduced accumulated film stress in several ${ }^{60}$ material systems. Difficulties found in manual polishing, alongside the observed optical artifacts with $40 \mu \mathrm{m}$ thick MLL deposited in pure Ar were overcome with the addition of $\mathrm{N}_{2}$, leading to MLLs that could now be deposited over $70 \mu \mathrm{m}$ thick, almost a factor of $2 \times$ increase. The addition of $\mathrm{N}_{2}$ appears to produce a silicon nitride layer resulting in a slightly different required section width, as well as a very minor decrease in total efficiency for flat MLL. ${ }^{26}$

In order to get over the $100 \mu \mathrm{m}$ MLL deposition thickness, a new aluminum-silicon based material composition has been selected as a replacement for silicon as the spacer layer. At X-ray wavelengths, aluminum is optically similar to silicon. In order to explore this new material, a $102 \mu \mathrm{m}$ thick MLL with an outermost zone width of $4 \mathrm{~nm}$ and a focal length of $9.6 \mathrm{~mm}$ at a photon energy of $12 \mathrm{keV}$ aiming to a $10 \mathrm{~nm}$ focus was fabricated. The MLL is comprised of zones $632-15,802$ of alternating $\mathrm{WSi}_{2}$ and $\mathrm{Al}-\mathrm{Si}$ layers. The deposited layers correspond to the outer $80 \%$ of a half structure MLL, intended to be used for focusing in an off-axis geometry. This thickness is roughly double the thickness reported ${ }^{4,45}$ in previous fabrication attempts.

The device was deposited in the MLL deposition system at Brookhaven National Laboratory using four out of nine available cathodes. ${ }^{61}$ The $\mathrm{WSi}_{2}$ targets are identical in composition to previous work. The Aluminum targets included roughly $5 \%$ silicon (by weight). The process gas consisted of $90 \% \mathrm{Ar}$ and $10 \% \mathrm{~N}_{2}$ with deposition pressure held at 4 milliTorr using the standard upstream control feedback system. Cathode power for all materials was $350 \mathrm{~W}$. Due to the rather large aspect ratios targeted for this lens (total deposition thickness over $100 \mu \mathrm{m}$, section width just under $10 \mu \mathrm{m}$ ), it was expected that this lens would warp and bow if left as a free-standing structure, so the sectioning process included bonding the MLL to a diamond plate. After the deposition, the substrate and accompanying multilayer were sandwiched and diced into $2 \times 2 \mathrm{~mm}$ squares. One face was polished using standard methods, and then this face was bonded to a $50 \mu \mathrm{m}$ thick diamond plate. After bonding, the entire MLL was thinned to a section width of about $8 \mu \mathrm{m}$ by grinding and polishing the face opposite the diamond plate. The lens remains permanently bonded to the diamond plate during all X-ray experimentation. About $2 \%$ of the incident beam will be attenuated by the diamond but no other significant effect on measurements is expected by the diamond plate given its small thickness, low absorption and overall uniformity. SEM images of the sectioned MLL in Figure 2 show good layer contrast throughout the entire deposition.

The intent for this test structure is to explore or verify whether this new material is compatible with

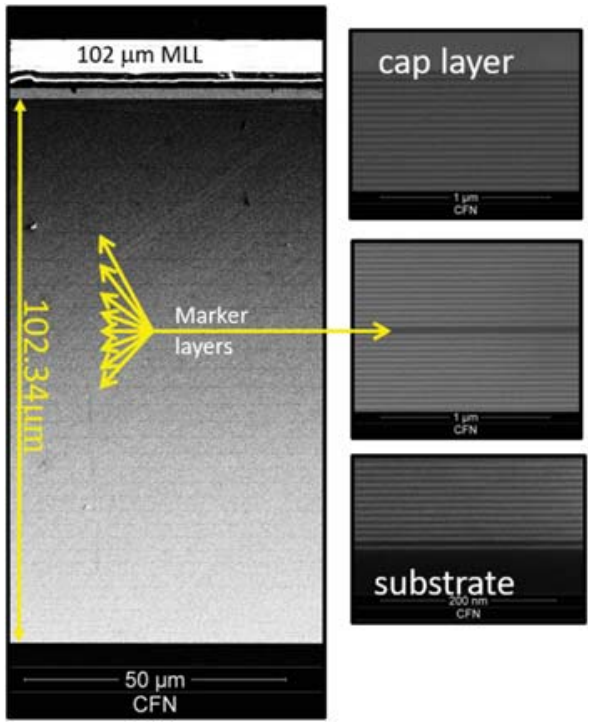

Figure 2. SEM images of $102 \mu \mathrm{m}$ thick $\mathrm{WSi}_{2} / \mathrm{Al}-\mathrm{Si}$ MLL with $3 \mu \mathrm{m}$ cap layer. The images reveal high contrast and smooth deposition throughout the entire structure. 


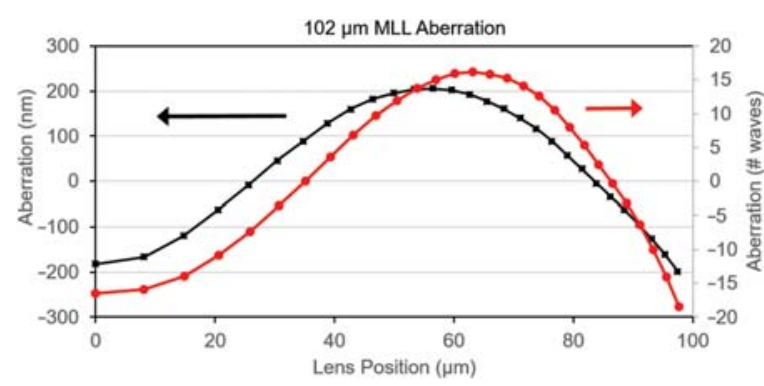

Figure 3. Layer placement error of the $102 \mu \mathrm{m}$ thick $\mathrm{WSi}_{2} / \mathrm{Al}_{5} \mathrm{Si} \mathrm{MLL}$ plotted in units of nm (black squares) and number of waves (red circles). Intended only for exploration of large aperture MLL fabrication and not ultimate focusing, only the first iteration of this MLL was deposited. Multiple iterations would be required in order to produce a diffractionlimited MLL.

significantly thicker multilayer deposition (over $100 \mu \mathrm{m}$ ) while maintaining sufficiently low interfacial roughness, not to examine limits for focusing performance. As such, this structure contained marker layers, but only one coating iteration took place. The significant layer placement error in the as-deposited multilayer plotted below in Figure 3. In order to reduce the layer placement error, deposition parameters will need to be corrected using the growth error measured on this first multilayer as feedback. When any single MLL design parameter is changed (total thickness, materials, focal length, or number of layers) the accumulated layer placement error is rather large with the 1st iteration. This error can be attributed to multiple, complicated phenomenon during the deposition including target erosion, nonlinear deposition rates, or slight changes in the flux plume distribution. However, past experience has demonstrated that overall repeatability of the deposition equipment allows successive iterations of corrective deposition in order to greatly reduce this error. For comparison, a $43 \mu \mathrm{m}$ MLL with 6,510 layers required 4 iterations to reduce placement error from $200 \mathrm{~nm}$ down to around $4 \mathrm{~nm}$.
Multilayers used for X-ray focusing require (almost) entirely amorphous substrates. Crystalline or nanocrystalline formation may cause undesirable reflections and commonly leads to interlayer roughness resulting in eventual loss of optical contrast, or nonlinear deposition rates that may be difficult to account for. As Aluminumbased materials are not commonly used in thin-film based $\mathrm{X}$-ray optics, the possibility of crystalline formation or propagation of structure and roughness to the surface was assessed by two techniques. The top surface of the $\mathrm{Al}_{5} \mathrm{Si}$ cap layer was measured for roughness with a visible light microscope interferometer. Over a field of view of 180 $\mu \mathrm{m} \times 130 \mu \mathrm{m}$, RMS roughness is $3.2 \AA$. A false-color height map of the measurement is shown in Figure 4(a). The $\mathrm{WSi}_{2} / \mathrm{Al}-\mathrm{Si}$ sectioned MLL was also investigated in a simple $\Theta / 2 \Theta$ diffraction geometry at $12 \mathrm{keV}$ to scan for diffraction peaks that might indicate crystalline formation. The diffraction measurement is seen in Figure 4(b).

Mechanical deformation, such as warping, delamination and kinks in the lens can be caused by internal film stresses, polishing and sectioning processes, dicing, and the gluing process. The sectioned lens under test has a complete length of $2.7 \mathrm{~mm}$. A study of the lens at beamline $1 \mathrm{BM}$ at the Advanced Photon Source revealed that the lens has defects along many, but not all regions of this length. In the experiment ${ }^{62}$ by Kubec et al., monochromatic beam at $12 \mathrm{keV}$ illuminates the MLL which was mounted on the Prototype MLL microscope $e^{63-65}$ in horizontal diffraction geometry. Absorbing slits were positioned to cover all but the actual structure. The MLL was rocked around a small set of tilt angles while images were collected in the far field with imaging detectors.

Orthogonal views of the first focusing orders are presented in Figure 5. For these views, the horizontal axis represents the angle of the lens with respect to the beam, and the vertical axis represents location along the lens. For these data sets, each image in Figure 5 represents the

\section{$102 \mu \mathrm{m} \mathrm{WSi}{ }_{2} / \mathrm{Al}-\mathrm{Si}$ MLL Microstructure}

Al-Si Cap Layer surface measurement

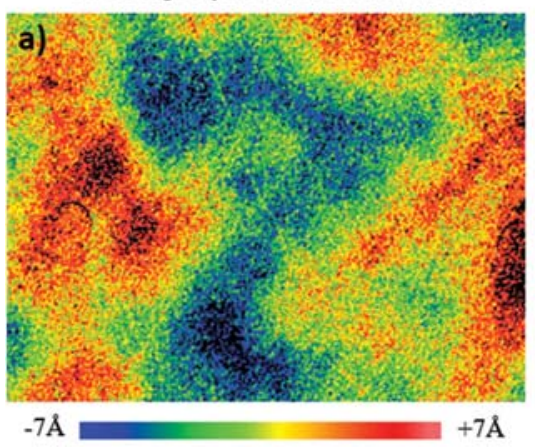

Diffraction

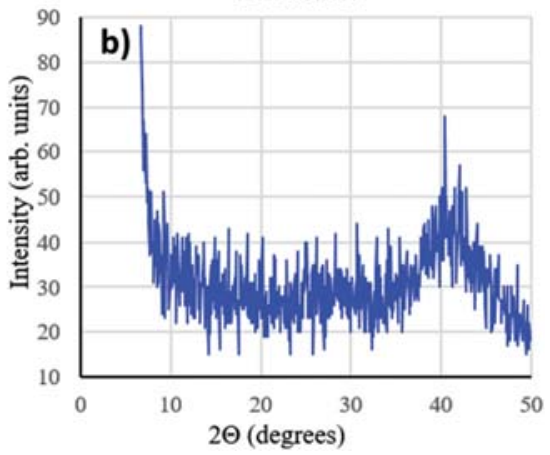

Figure 4. Measurement of properties of Al-Si. (a) A visible-light interferometric microscopy roughness measurement of the topmost surface of the Al-Si cap layer. RMS roughness is $3.2 \AA$ over a field of view of $180 \mu \mathrm{m}$ by $130 \mu \mathrm{m}$. (b) X-ray reflectivity measurement at $12 \mathrm{keV} 102 \mu \mathrm{m} \mathrm{WSi} / \mathrm{Al}_{-} \mathrm{Si}$ MLL structure. Only a broad peak is seen at $2 \theta=40^{\circ}$ which indicates a feature size of $0.15 \mathrm{~nm}$ within the structure. 

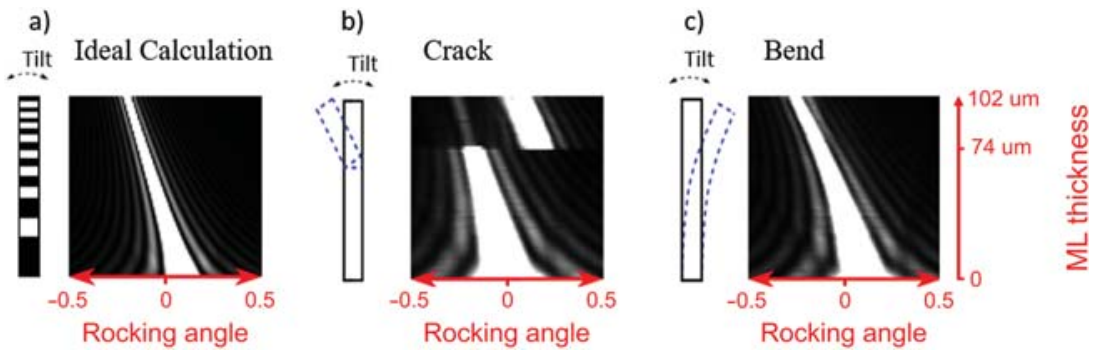

Figure 5. Orthogonal views for (a) ideal MLL, (b) a lens with a kink or discontinuity and (c) a gradually bent lens.

compilation of an entire series of frame grabs from the detector at discrete angles. The results of coupled wave theory calculations ${ }^{62}$ for an ideal lens are presented in Figure 5(a). The calculation shows a smooth progression with tilted angle lacking any discontinuities. Part (b) shows a discontinuity in the angle of the lens at about the $74 \mu \mathrm{m}$ region. This discontinuity may be explained by a kink in the lens resulting from accumulated film stress. The gradual bend in part (c) could be due to stresses from sectioning, and is likely compounded by the rather large aspect ratio for this structure. Utilizing a lower density absorber instead of $\mathrm{WSi}_{2}$ would result in a thicker section width, reducing this bending. On the other hand, this bending could be useful as a method to produce a wedged structure from an initially flat or planar MLL deposition. Deposition of a thin-film on the side of a sectioned MLL to induce stress-based curvature, thus leading to a wMLL, has been reported. ${ }^{41}$ Multiple locations across the lens were reported to contain cracks, bends, or other defects; however, one region at the end of the $2.7 \mathrm{~mm}$ long lens was defect free. This region was characterized by measuring efficiency of the negative and positive first orders. Negative and positive first orders were found to have $14.2 \%$ and $13.0 \%$ efficiencies, ${ }^{66}$ respectively. A focus size of $25 \mathrm{~nm}^{67}$ was determined by ptychographic phase retrieval ${ }^{68,69}$ at $12 \mathrm{keV}$. A lens with the same optical parameters but lacking any layer placement error would produce a diffraction-limited focus size of about $10 \mathrm{~nm}$.

\section{INCREASING EFFICIENCY WITH wMLL}

Flat (or tilted) MLLs lose efficiency when spatial resolution approaches around $10 \mathrm{~nm}$ due to dynamical diffraction effects. ${ }^{27}$ To approach single-nanometer focus size with the potential for efficiency over $80 \%$ for a single lens (thus, efficiency when point focusing with two lens is comfortably greater than $50 \%$ ), wMLLs with progressively tilted zones such as seen in Figure 1(g) are required. The optimum depth along the optical axis of an MLL required for high efficiency is, with the material systems and energy ranges of interest at present, no larger than about $20 \mu \mathrm{m}$. If lower density materials are introduced in the future, section depth would still remain in the 100-200 $\mu \mathrm{m}$ range, even for $100 \mathrm{keV}$ radiation. Because focal lengths (several $\mathrm{mm}$ ) are significantly larger than the section width, a linear gradient within the optic is sufficient. We employ a profile-coating technique ${ }^{70}$ to produce wMLL, where a figured mask produces a steep thickness gradient along the axis of substrate travel within the deposition system. It should be noted that growth of non-linear gradients such as parabolic or elliptical shapes which can be used for sub $1 \mathrm{~nm}$ focusing optics shown in Figure 1(h) are achievable with the same coating technique. Details on geometry considerations are discussed in more detail elsewhere. ${ }^{26,59}$ The actual change in the deposition rate gradient along the optical axis is twice the focal length of the layer sequence, and must match the designed focal length of the MLL with precision that keeps the focus well within the depth of focus of the optic. However, because the layer sequence depends on the product of both wavelength and focal length, and the deposition technique produces a gradient that is, by default, additively uniform throughout the entire stack, there will always be some wavelength with which any built-in deposition gradient (even the wrong one) will match. So just as with flat or tilted MLLs, as long as the layer placement within the wMLL is adequate, the proper wavelength can be selected to match the focal length that is dictated by the in-plane deposition gradient. This means that many wMLL sections can be extracted from a single coating sample, but the optimum energy and focal length for each section will differ depending on the deposition rate gradient along the optical axis.

Calculations on the original proof-of-concept wMLL fabricated at the Advanced Photon Source ${ }^{36}$ with 1,588 layers of $\mathrm{WSi}_{2} / \mathrm{Si}$, indicate that a section $30 \mu \mathrm{m}$ thick should produce a focal spot size of $5.5 \mathrm{~nm}$ at $82.1 \mathrm{keV}$ (the unusual energy is due to the combination of wedge angle and layer placement) with an efficiency of $30 \%$. The energy tolerance was estimated to be $\pm 10 \% .^{71}$ Just like other optics, wMLL structures have an energy acceptance range, where, according to dynamical diffraction theory, ${ }^{27}$ the focusing performance is not significantly degraded. Lower-energy devices will exhibit a proportionally smaller energy bandwidth. A series of three wMLLs produced using the MLL deposition system at Brookhaven National Laboratory ${ }^{72}$ were selected to study the relationship between efficiency and energy. The first two were 


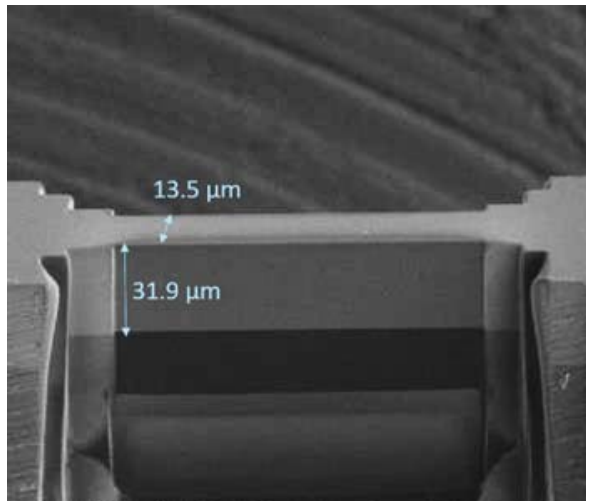

Figure 6. SEM image of wMLL B-13.5, illustrating an MLL deposition thickness of $31.9 \mu \mathrm{m}$ and a section width of $13.5 \mu \mathrm{m}$.

$\mathrm{WSi}_{2} / \mathrm{Si}$ based with aperture size $25 \mu \mathrm{m}$ and $39 \mu \mathrm{m}$ thick respectively. While the third one is identical in structure to the $25 \mu \mathrm{m}$ MLL but is fabricated with $\mathrm{WSi}_{2} / \mathrm{Si}-\mathrm{Al}$ in order to start introducing wedging with this new material system. All MLL were deposited with four cathodes, at 4.0 milliTorr in a $5 \% \mathrm{~N}_{2} / 95 \%$ Ar process gas. The parameters are summarized in Figure 8. Details on the deposition process, masking apparatus, and focusing X-ray measurements on similar lens can be found elsewhere. ${ }^{73}$ The first lens was sectioned at three different locations, thus providing three different lenses with distinct focal-length/energy products. After deposition, each lens was patterned, rough sectioned using reactive ion etching, and then the faces of the etched lens were put through a final thinning and polishing process using a focused ion-beam (FIB) milling instrument. An example of one of the lens is shown below in Figure 6.

Diffraction measurements as a function of rocking angle of all 5 wMLL lens segments were made at APS beamline 1-BM ${ }^{74}$ using the same prototype MLL microscope as the previous work. The lens was aligned to diffract onto a Pilatus $100 \mathrm{~K}$ area detector ${ }^{75}$ placed $900 \mathrm{~mm}$ downstream of the lens. Both positive (focusing) and negative (defocusing) first order diffraction patterns were recorded in the far-field. The orthogonal views provide an easy way to understand the characteristics of diffraction as function of layer thicknesses and rocking angles. A series of three measurements at $13.5 \mathrm{keV}, 16 \mathrm{keV}$, and $19 \mathrm{keV}$ are presented in Figure 7 of the wMLL $25 \mu \mathrm{m}-11.1$. The prominent slope in the -1 st orders indicates that only a small region within the wMLL are excited at any given energy and the location within the optic is dependent on rocking angle. In this way, wMLL function much like a tilted or flat MLL when de-focusing. When the wMLL is aligned properly, horizontally, the +1 st orders show highly uniform focusing efficiency. Comparison of the observed slope of the +1 st order focusing region at the three measured energies indicates that this wMLL matches $16 \mathrm{keV}$ best.
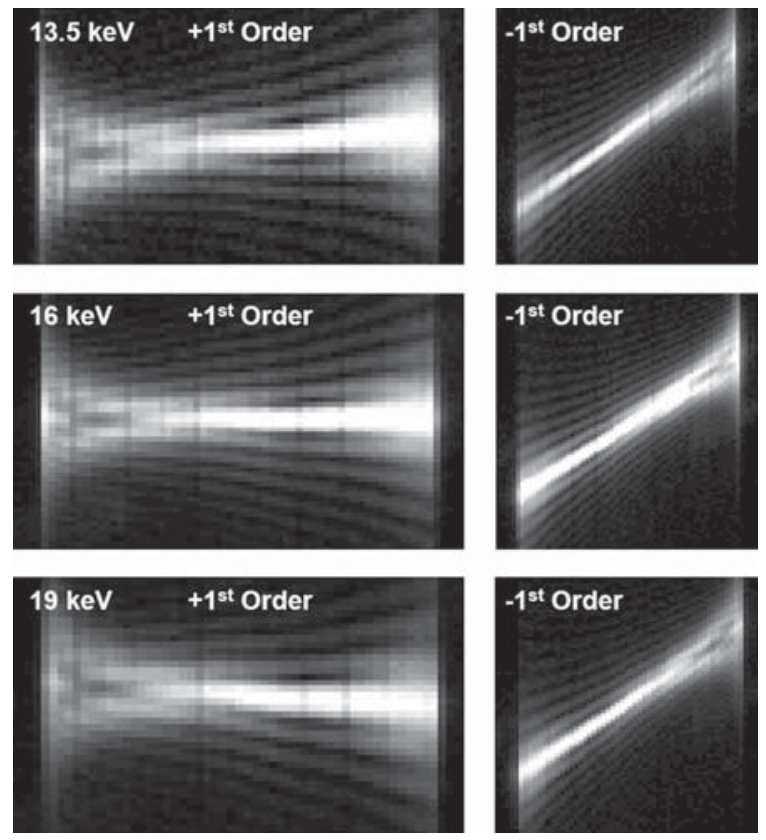

Figure 7. Positive first order and negative first order diffraction for the lens A-11.1. The horizontal axis represents horizontal distance along the detector, and the vertical axis represents rocking angle. An ideal wMLL would have perfectly horizontal diffraction intensity in the first order. A slight horizontal tilt can be seen at $19 \mathrm{kev}$ and $13.5 \mathrm{keV}$. The wedging and layer sequence in this structure are optimized for $16 \mathrm{keV}$. Vertical banding comes from marker layers inserted in the stack for layer position metrology.

Diffraction patterns for all five wMLLs were measured in a similar manner, and integrated intensity of the +1 st order peak at the optimum rocking angle is summed and plotted for a set of energies ranging from $13 \mathrm{keV}$ up to $19 \mathrm{keV}$. Total efficiency versus energy for all five lenses is plotted in Figure 8 below. The ideal section width for $\mathrm{WSi}_{2} / \mathrm{Si}$ is about $9.5 \mu \mathrm{m}$ and $15 \mu \mathrm{m}$ at $13 \mathrm{kev}$, and $20 \mathrm{keV}$, respectively. The ideal section width for $\mathrm{WSi}_{2} / \mathrm{Al}-\mathrm{Si}$ is $10.1 \mu \mathrm{m}$ and $16 \mu \mathrm{m}$ at $13 \mathrm{keV}$ and $20 \mathrm{keV}$, respectively. Again, four of the five lenses have identical design parameters and aperture. Three of these are $\mathrm{WSi}_{2} / \mathrm{Si}$, and the fourth is $\mathrm{WSi}_{2} / \mathrm{Al}-\mathrm{Si}$. The fifth lens is also $\mathrm{WSi}_{2} / \mathrm{Si}$, but with a larger aperture. Maximum efficiency is noted for the $25 \mu \mathrm{m} \mathrm{WSi}{ }_{2} / \mathrm{Si}$ with $11 \mu \mathrm{m}$ section width of just over $35 \%$ at $17 \mathrm{keV}$. Maximum efficiency for a second lens from the set with an almost identical section width $(11.1 \mu \mathrm{m})$ was slightly lower, with a maximum of about $30 \%$ between $18-19.5 \mathrm{keV}$, with one outlier data in the series. Efficiency for the third lens was significantly lower at $15 \%$, also highest in $19 \mathrm{keV}$ range. This is attributed to a much smaller section width of $7 \mu \mathrm{m}$. The fourth lens in the set, where the silicon spacer layer has been substituted for $\mathrm{Al}-\mathrm{Si}$, has maximum efficiency of $\sim 25 \%$ around the $14 \mathrm{keV}$ range. The fifth lens, a $31.9 \mu \mathrm{m}$ aperture $\mathrm{WSi}_{2} / \mathrm{Si}$ wMLL, has 


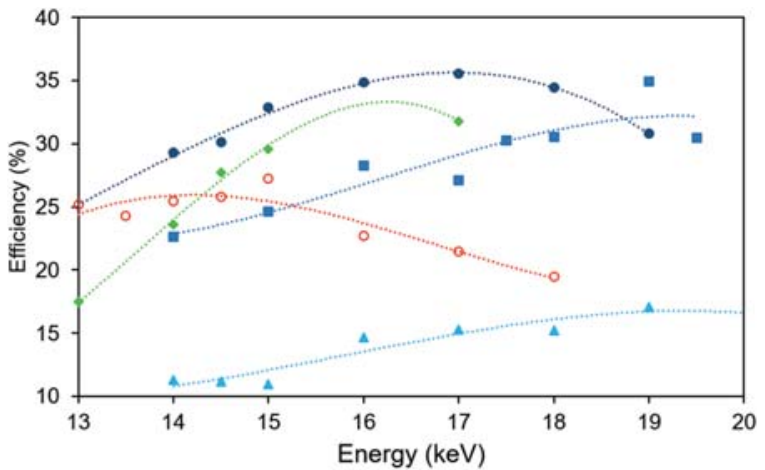

\begin{tabular}{|c|c|c|c|c|c|}
\hline Tag & Lens & $\begin{array}{l}\text { Coating } \\
\text { Run }\end{array}$ & $\begin{array}{c}\text { Aperture } \\
(\mu \mathrm{m})\end{array}$ & $\begin{array}{c}\text { Section } \\
\text { Width }(\mu \mathrm{m})\end{array}$ & Material \\
\hline$\bullet$ & $25 \mu m-11$ & A & 25 & 11 & WSi ${ }_{2} / \mathrm{Si}_{\mathrm{x}} \mathrm{N}_{\mathrm{x}}$ \\
\hline - & $25 \mu m-11.1$ & A & 25 & 11.1 & WSi $/$ Si $_{x} \mathrm{~N}_{x}$ \\
\hline$\Delta$ & $25 \mu m-7$ & A & 25 & 7 & WSi ${ }_{2} / \mathrm{Si}_{\mathrm{x}} \mathrm{N}_{\mathrm{x}}$ \\
\hline$\cdot$ & $31.9 \mu \mathrm{m}-13.5$ & B & 31.9 & 13.5 & WSi ${ }_{2} / \mathrm{Si}_{x} \mathrm{~N}_{\mathrm{x}}$ \\
\hline$\circ$ & 25um-9 & C & 25 & 9 & $\mathrm{WSi}_{2} /(\mathrm{Si}-\mathrm{Al}) \mathrm{N}_{2}$ \\
\hline
\end{tabular}

Figure 8. Measured wMLL efficiency dependence on energy for a range of energies measured at 1-BM at the APS. Five section lenses were tested. Maximum efficiency of $35 \%$ was observed. For chromatic optics, wMLL efficiency is rather insensitive to change in energy of several kilovolts. This observation corroborates other reported work, and suggests that during use wMLLs may allow some range of energy tunability.

maximum efficiency of about $30 \%$ at $17 \mathrm{keV}$, the highest energy measured with this lens.

\section{CONCLUSIONS}

Larger MLL deposition thicknesses for increased aperture are obtained through deposition process optimization and the introduction of new material systems. $\mathrm{WSi}_{2} / \mathrm{Si}$ MLLs that were fabricated with $\mathrm{N}_{2}$ reactive sputtering are currently in use at the $\mathrm{HXN}^{76}$ beamline at NSLS-II. These optics have an aperture size of $43 \mu \mathrm{m} \times 53 \mu \mathrm{m}$, producing a focus below $20 \times 20 \mathrm{~nm}^{2}$. An MLL with a world-leading aperture size over $100 \mu \mathrm{m}$ was tested ${ }^{62}$ at wavelength, and shown to possess excellent optical contrast and sharp interfaces. These MLLs replace the nitrogen reactivelysputtered silicon spacer layer with Al-Si. Proportionally, substitution of $\mathrm{Si}$ with $\mathrm{Al}-\mathrm{Si}$ is calculated to reduce the efficiency by just a few percent at the lower range of energies, and is insignificant at energies over $20 \mathrm{keV}$. The exploration of reactive deposition, and new material systems led to an almost factor of $10 \times$ increase in total aperture to lenses that exceed $102 \mu \mathrm{m}$. The $\mathrm{WSi}_{2} / \mathrm{Al}-\mathrm{Si}$ material system currently is the largest aperture MLL produced. Thicknesses in the $100 \mu \mathrm{m}$ range do not appear to be the upper limit, and larger sizes should be explored. Also, this first large area MLL is not ideal in several respects, most notably, in that as the first iteration, layer placement error is very large.

wMLLs, for utmost focusing and optical efficiency, are fabricated and sectioned. The energy dependence of these
wMLL samples is about 35\%, and the energy range is sufficiently wide that a single wMLL sample may be used for a limited range of energy scanning. Currently wMLL aperture lags about $2 \times$ or $3 \times$ behind flat MLL mainly due to the reduced efficiency in the wMLL masking as well as a desire to produce usable wMLL optics, not simply chase aperture size. Recent efforts concentrated on integrating the developed process for large aperture $\mathrm{WSi}_{2} / \mathrm{Al}-\mathrm{Si}$ based flat MLL into wedged structures. If diffraction-limited wMLLs with apertures approaching $100 \mu \mathrm{m}$ become reality, attention can be directed back towards making further gains in aperture size. Looking forward, bonding of two linear orthogonally pre-aligned individual MLLs (essentially creating a drop-in MLL replacement ${ }^{77,78}$ for a zone plate) will make their incorporation into microscopy systems straight forward, enabling much broader applications of these nanofocusing optics. While the many circular wire multilayer zone plate efforts have, at least for now, reached limits due to a variety of reasons, these would be the most convenient optics for use due their ability to directly replace conventional zone plates. Reaching below $10 \mathrm{~nm}$ focus requires properly tapered zones, so the multilayer zone plate would require a wedged, or curved substrate such as a tapered wire or a section of a sphere. This is a natural progression of the technology and hopefully will become reality in the future.

Acknowledgments: The authors would like to acknowledge Adam Kubec for his help on the characterization of the 102 microns MLL and Michael Wojcik for his help on the experiments carried at the 1BM beamline at the Advanced Photon Source at Argonne National Laboratory. Work at the Argonne National Laboratory was supported by the U.S. DOE under Contract No. DE-AC0206CH11357. Work at Brookhaven National Laboratory was supported by the Department of Energy, Office of Basic Energy Sciences under contract DE-SC00112704. The Advanced Photon Source and The National Synchrotron Light Source-II are Office of Science User Facilities operated for the U.S. Department of Energy (DOE) Office of Science by Argonne National Laboratory, and Brookhaven National Laboratory, respectively.

\section{References and Notes}

1. X. Huang, H. Yan, E. Nazaretski, R. Conley, N. Bouet, J. Zhou, K. Lauer, L. Li, D. Eom, D. Legnini, R. Harder, I. K. Robinson, and Y. S. Chu, Scientific Reports 3, 3562 (2013).

2. E. Nazaretski, X. Huang, H. Yan, K. Lauer, R. Conley, N. Bouet, J. Zhou, W. Xu, D. Eom, D. Legnini, R. Harder, C.-H. Lin, Y.-S. Chen, Y. Hwu, and Y. S. Chu, Rev. Sci. Instrum. 85, 033707 (2014).

3. E. Nazaretski, H. Yan, K. Lauer, N. Bouet, X. Huang, W. Xu, J. Zhou, D. Shu, Y. Hwu, and Y. S. Chu, Journal of Synchrotron Radiation 24, 1113 (2017).

4. A. J. Morgan, M. Prasciolu, A. Andrejczuk, J. Krzywinski, A. Meents, D. Pennicard, H. Graafsma, A. Barty, R. J. Bean, M. Barthelmess, D. Oberthuer, O. Yefanov, A. Aquila, H. N. Chapman, and S. Bajt, Scientific Reports 5, 9892 (2015). 
5. S. Bajt, M. Prasciolu, H. Fleckenstein, M. Domaracký, H. N. Chapman, A. J. Morgan, O. Yefanov, M. Messerschmidt, Y. Du, K. T. Murray, V. Mariani, M. Kuhn, S. Aplin, K. Pande, P. Villanueva-Perez, K. Stachnik, J. P. J. Chen, A. Andrejczuk, A. Meents, A. Burkhardt, D. Pennicard, X. Huang, H. Yan, E. Nazaretski, Y. S. Chu, and C. E. Hamm, Nature Light: Science and Applications 7, 17162 (2017).

6. K. Yamauchi, H. Mimura, T. Kimura, H. Yumoto, S. Handa, S. Matsuyama, K. Arima, Y. Sano, K. Yamamura, K. Inagaki, H. Nakamori, J. Kim, K. Tamasaku, Y. Nishino, M. Yabashi, and T. Ishikawa, Journal of Physics: Condensed Matter 23, 394206 (2011).

7. C. G. Schroer, O. Kurapova, J. Patommel, P. Boye, J. Feldkamp, B. Lengeler, M. Burghammer, C. Riekel, L. Vincze, A. van der Hart, and M. Kuchler, Appl. Phys. Lett. 87, 124103 (2005).

8. K. Burkhard, T. Pierre, G. Alessandra, and K. Maya, Journal of Physics: Condensed Matter 23, 083002 (2011).

9. J. Kirz, C. Jacobsen, and M. Howells, Quarterly Reviews of Biophysics 28, 33 (1995).

10. I. Mohacsi, I. Vartiainen, B. Rösner, M. Guizar-Sicairos, V. A. Guzenko, I. McNulty, R. Winarski, M. V. Holt, and C. David, Scientific Reports 7, 43624 (2017).

11. C. Tsung-Yu, C. Yu-Tung, W. Cheng-Liang, I. M. Kempson, L. Wah-Keat, Y. S. Chu, Y. Hwu, and G. Margaritondo, Opt. Express 19, 19919 (2011).

12. J. Vila-Comamala, S. Gorelick, E. Färm, C. M. Kewish, A. Diaz, R. Barrett, V. A. Guzenko, M. Ritala, and C. David, Opt. Express 19, 175 (2011).

13. S.-R. Wu, Y. Hwu, and G. Margaritondo, Materials 5, 1752 (2012).

14. K. Parfeniukas, J. Rahomäki, S. Giakoumidis, F. Seiboth, F. Wittwer, C. G. Schroer, and U. Vogt, Microelectron. Eng. 152, 6 (2016).

15. G. Aquilanti, L. Vaccari, J. R. Plaisier, and A. Goldoni, Synchrotron Radiation: Basics, Methods and Applications, edited by S. Mobilio, F. Boscherini, and C. Meneghini, Springer Berlin Heidelberg, Berlin, Heidelberg (2015), pp. 65-104.

16. K. Saitoh, K. Inagawa, K. Kohra, C. Hayashi, A. Iida, and N. Kato, Japanese Journal of Applied Physics 27, L2131 (1988).

17. R. M. Bionta, K. M. Skulina, and J. Weinberg, Appl. Phys. Lett. 64, 945 (1994)

18. B. Kaulich, Advances in X-ray/EUV Optics and Components II 3449, 108 (1998).

19. R. M. Bionta, Appl. Phys. Lett. 51, 725 (1987).

20. D. Rudolph, B. Niemann, and G. Schmahl, Proc. SPIE, High Resolution Soft X-Ray Optics 316 (1982).

21. F. Döring, A. L. Robisch, C. Eberl, M. Osterhoff, A. Ruhlandt, T. Liese, F. Schlenkrich, S. Hoffmann, M. Bartels, T. Salditt, and H. U. Krebs, Opt. Express 21, 19311 (2013).

22. T. Koyama, H. Takano, S. Konishi, T. Tsuji, H. Takenaka, S. Ichimaru, T. Ohchi, and Y. Kagoshima, Rev. Sci. Instrum. 83, 013705 (2012).

23. T. Koyama, T. Tsuji, H. Takano, Y. Kagoshima, S. Ichimaru, T. Ohchi, and H. Takenaka, 10th International Conference on X-ray Microscopy (2011), p. 1365.

24. M. Mayer, C. Grévent, A. Szeghalmi, M. Knez, M. Weigand, S. Rehbein, G. Schneider, B. Baretzky, and G. Schütz, Ultramicroscopy 111, 1706 (2011).

25. M. Mayer, K. Keskinbora, C. Grevent, A. Szeghalmi, M. Knez, M. Weigand, A. Snigirev, I. Snigireva, and G. Schutz, Journal of Synchrotron Radiation 20, 433 (2013).

26. H. Yan, R. Conley, N. Bouet, and Y. S. Chu, J. Phys. D: Appl. Phys. 47, 263001 (2014)

27. H. F. Yan, J. Maser, A. Macrander, Q. Shen, S. Vogt, G. B. Stephenson, and H. C. Kang, Physical Review B 76, 115438 (2007).

28. H. Yan, J. Maser, H. C. Kang, A. Macrander, and B. Stephenson, Advances in X-Ray/EUV Optics and Components II 7077, 70770Q (2008).
29. H. F. Yan, Physical Review B 79 (2009).

30. J. Maser, G. B. Stephenson, S. Vogt, Y. Wenbing, A. Macrander, H. C. Kang, L. Chian, and R. Conley, Advances in X-ray/EUV Optics and Components II 5539, 185 (2004).

31. C. Liu, R. Conley, A. T. Macrander, J. Maser, H. C. Kang, M. A Zurbuchen, and G. B. Stephenson, J. Appl. Phys. 98,113519 (2005).

32. H. C. Kang, J. Maser, G. B. Stephenson, C. Liu, R. Conley, A. T. Macrander, and S. Vogt, Phys. Rev. Lett. 96, 127401 (2006).

33. H. C. Kang, H. Yan, R. P. Winarski, M. V. Holt, J. Maser, C. Liu, R. Conley, S. Vogt, A. T. Macrander, and G. B. Stephenson, Appl. Phys. Lett. 92, 221114 (2008).

34. C. Liu, R. Conley, J. Qian, C. Kewish, A. Macrander, J. Maser, H. Kang, H. Yan, and G. Stephenson, Nuclear Instruments and Methods in Physics Research Section A: Accelerators, Spectrometers, Detectors and Associated Equipment 582, 123 (2007).

35. R. Conley, C. Liu, A. Macrander, H. Yan, H. Kang, J. Maser, and G. B. Stephenson, U.S.A. Patent No. 9,082,522 (2015).

36. R. Conley, C. Liu, J. Qian, C. M. Kewish, A. T. Macrander, H. Yan, H. C. Kang, J. Maser, and G. B. Stephenson, Rev. Sci. Instrum. 79, 053104 (2008).

37. T. Koyama, H. Takenaka, S. Ichimaru, T. Ohchi, T. Tsuji, H. Takano, and Y. Kagoshima, 10th International Conference on $X$-ray Microscopy, edited by I. McNulty, C. Eyberger, and B. Lai, Amer Inst Physics, Melville (2011), Vol. 1365, pp. 24-27.

38. S. Braun, A. Kubec, M. Menzel, S. Niese, P. Krüger, F. Seiboth, J. Patommel, and C. Schroer, Journal of Physics: Conference Series 425, 052019 (2013).

39. T. Liese, V. Radisch, I. Knorr, M. Reese, P. Gro $\beta$ mann, K. Mann, and H. U. Krebs, Appl. Surf. Sci. 257, 5138 (2011).

40. T. Liese, V. Radisch, and H. U. Krebs, Rev. Sci. Instrum. 81, 073710 (2010).

41. S. Niese, P. Krüger, A. Kubec, R. Laas, P. Gawlitza, K. Melzer, S. Braun, and E. Zschech, Thin Solid Films 571, Part 2, 321 (2014).

42. M. Prasciolu, A. F. G. Leontowich, J. Krzywinski, A. Andrejczuk, H. N. Chapman, and S. Bajt, Optical Materials Express 5, 748 (2015).

43. L. Zhou, S. Yue, M. Li, P. Liu, and G. Chang, Nuclear Instruments and Methods in Physics Research Section A: Accelerators, Spectrometers, Detectors and Associated Equipment 806, 87 (2016).

44. X. Huang, H. Yan, E. Nazaretski, R. Conley, N. Bouet, J. Zhou, K. Lauer, L. Li, D. Eom, D. Legnini, R. Harder, I. K. Robinson, and Y. S. Chu, Scientific Reports 3 (2013).

45. A. J. Morgan, M. Prasciolu, A. Andrejczuk, J. Krzywinski, A. Meents, D. Pennicard, H. Graafsma, A. Barty, R. J. Bean, and M. Barthelmess, Scientific Reports 5 (2015).

46. T. Kozawa, T. Kachi, H. Kano, H. Nagase, N. Koide, and K. Manabe, J. Appl. Phys. 77, 4389 (1995).

47. H. Ibach, Surf. Sci. Rep. 29, 195 (1997).

48. E. Chason, B. W. Sheldon, L. B. Freund, J. A. Floro, and S. J. Hearne, Phys. Rev. Lett. 88, 156103 (2002).

49. J. A. Floro, P. G. Kotula, S. C. Seel, and D. J. Srolovitz, Phys. Rev. Lett. 91, 096101 (2003).

50. A.-L. Barabási and H. E. Stanley, Fractal Concepts in Surface Growth, Cambridge University Press, Cambridge, UK (1995).

51. D. B. Chrisey and G. K. Hubler, Pulsed Laser Deposition of Thin Films, Wiley-Interscience, New York (1994).

52. D. W. Hoffman and J. A. Thornton, Journal of Vacuum Science and Technology 20, 355 (1982).

53. H. Windischmann, Critical Reviews in Solid State and Material Sciences 17, 547 (1992).

54. C. Liu, R. Conley, and A. T. Macrander, SPIE Optics + Photonics 6317 (2006).

55. K. MacArthur, B. Shi, R. Conley, and A. T. Macrander, Appl. Phys. Lett. 99, 081905 (2011).

56. J. Röder and H. U. Krebs, Appl. Phys. A 90, 609 (2008).

57. R. Conley Jr., C. Liu, A. Macrander, H. Yan, H. Kang, J. Maser, and G. B. Stephenson, U.S. Patent No. PCT/US2010/060057 (2008). 
58. B. Shi, A. T. Macrander, J. Maser, R. Conley, and L. Assoufid, SPIE Optical Engineering + Applications, Advances in X-ray/EUV Optics and Components IX 9207, 920708 (2014).

59. R. Conley, N. Bouet, J. Zhou, H. Yan, Y. Chu, K. Lauer, J. Miller, L. Chu, and N. Jahedi, SPIE Optical Engineering + Applications 8502,850202 (2012).

60. D. L. Windt, Optical Engineering + Applications 6688, 66880R (2007).

61. R. Conley, N. Bouet, K. Lauer, M. Carlucci-Dayton, J. Biancarosa, L. Boas, J. Drannbauer, J. Feraca, and L. Rosenbaum, AIP Conference Proceedings 1365, 69 (2011).

62. A. Kubec, N. Kujala, R. Conley, N. Bouet, J. Zhou, T. M. Mooney, D. Shu, J. Kirchman, K. Goetze, J. Maser, and A. Macrander, Opt. Express 23, 27990 (2015).

63. D. Shu, Y. Hanfei, and J. Maser, U.S.A Patent No. 7,597,475 (2009).

64. E. Nazaretski, J. Kim, H. Yan, K. Lauer, D. Eom, D. Shu, J. Maser, Z. Pesic, U. Wagner, C. Rau, and Y. S. Chu, Rev. Sci. Instrum. 84, 033701 (2013).

65. S. Deming, N. Evgeny, K. Jungdae, Y. Hanfei, L. Kenneth, M. Brian, K. Dennis, M. Jörg, and S. C. Yong, Journal of Physics: Conference Series 463, 012029 (2013).

66. A. T. Macrander, A. Kubec, R. Conley, N. Bouet, J. Zhou, M. Wojcik, and J. Maser, Appl. Phys. Lett. 107, 081904 (2015).

67. A. Macrander, M. Wojcik, J. Maser, N. Bouet, R. Conley, and B. Lai, SPIE Optical Engineering + Applications 10389 (2017).

68. J. N. Clark and A. G. Peele, Appl. Phys. Lett. 99, 154103 (2011).

69. P. Thibault, M. Dierolf, O. Bunk, A. Menzel, and F. Pfeiffer, Ultramicroscopy 109, 338 (2009).
70. C. Liu, L. Assoufid, A. T. Macrander, G. E. Ice, and J. Z. Tischler, International Symposium on Optical Science and Technology, X-ray Mirrors, Crystals, and Multilayers II (2002), p. 4782.

71. H. Yan, H. C. Kang, J. Maser, A. T. Macrander, C. M. Kewish, C. Liu, R. Conley, and G. B. Stephenson, Nucl. Instrum. Methods Phys. Res. Sect. A-Accel. Spectrom. Dect. Assoc. Equip. 582, 126 (2007).

72. R. Conley, N. Bouet, J. Biancarosa, Q. Shen, L. Boas, J. Feraca, and L. Rosenbaum, SPIE Optical Engineering + Applications, Advances in X-ray/EUV Optics and Components IV 7448 (2009).

73. X. Huang, R. Conley, N. Bouet, J. Zhou, A. Macrander, J. Maser, H. Yan, E. Nazaretski, K. Lauer, R. Harder, I. K. Robinson, S. Kalbfleisch, and Y. S. Chu, Opt. Express 23, 12496 (2015).

74. A. Macrander, M. Erdmann, N. Kujala, S. Stoupin, S. Marathe, X. Shi, M. Wojcik, D. Nocher, R. Conley, J. Sullivan, K. Goetze, J. Maser, and L. Assoufid, AIP Conference Proceedings 1741, 030030 (2016).

75. P. Kraft, A. Bergamaschi, C. Broennimann, R. Dinapoli, E. F. Eikenberry, B. Henrich, I. Johnson, A. Mozzanica, C. M. Schlepütz, P. R. Willmott, and B. Schmitt, Journal of Synchrotron Radiation 16, 368 (2009)

76. Y. Chu, H. Yan, E. Nazaretski, S. Kalbfleisch, X. Huang, K. Lauer, and N. Bouet, SPIE Newsroom (2015), DOI 10.1117/2.1201508.006068.

77. E. Nazaretski, W. Xu, N. Bouet, J. Zhou, H. Yan, X. Huang, and Y. Chu, Appl. Phys. Lett. 108, 261102 (2016).

78. X. Huang, W. Xu, E. Nazaretski, N. Bouet, J. Zhou, Y. S. Chu, and H. Yan, Opt. Express 25, 8698 (2017).

Received: 22 December 2017. Accepted: 8 May 2018. 